Ophthalmologe 2018 $\cdot 115: 202$ https://doi.org/10.1007/s00347-018-0668-2 Online publiziert: 15 . Februar 2018 (c) Springer Medizin Verlag $\mathrm{GmbH}$, ein Teil von Springer Nature 2018

CrossMark

Berufsverband der Augenärzte Deutschlands e.V. ${ }^{1}$ Deutsche Ophthalmologische Gesellschaft $^{2}$

'Berufsverband der Augenärzte Deutschlands e.V. (BVA), Düsseldorf, Deutschland

${ }^{2}$ Deutsche Ophthalmologische Gesellschaft (DOG), München, Deutschland

\title{
Stellungnahme von BVA und DOG zu Augenuntersuchung bei Patienten mit hohen Sehfehlern
}

\section{Stand Januar 2018}

Patienten mit hohen Sehfehlern leiden deutlich häufiger an behandlungsbedürftigen Augenkrankheiten als Patienten ohne oder mit geringeren Sehfehlern. Unter anderem sind folgende Krankheiten zu nennen:

- Myopie> 6 dpt: myope chorioidale Neovaskularisation, foveale Retinoschisis mit oder ohne Makulaforamen, Amotio retinae, Glaukom,

- Hyperopie > 6 dpt: Winkelblockglaukom v. a. bei zunehmender Katarakt,

- Astigmatismus > 4 dpt: Keratokonus, Pterygium, Zustand nach Keratoplastik.

Da bei diesen Erkrankungen häufig initial bzw. bei Verschlechterung nur leichte und untypische Beschwerden auftreten, äußern die Betroffenen oft zunächst nur den Wunsch nach einer neuen Brille.

Die Deutsche Ophthalmologische Gesellschaft (DOG) und der Berufsverband der Augenärzte Deutschlands (BVA) empfehlen deswegen den Augenärzten, vor allen Brillenverordnungen für Patienten mit den genannten hohen Sehfehlern mit entsprechenden Untersuchungen die oben genannten Krankheiten auszuschließen bzw. auf deren Verschlechterung zu kontrollieren und ggf. zu prüfen, ob nicht anstelle einer Brillenverordnung eine andere z. B. chirurgische oder medikamentöse Therapie erforderlich ist.

\section{Korrespondenzadresse}

Deutsche Ophthalmologische Gesellschaft

Deutsche Ophthalmologische Gesellschaft

(DOG)

Platenstr. 1, 80336 München, Deutschland geschaeftsstelle@dog.org

\section{Einhaltung ethischer Richtlinien}

Interessenkonflikt. Der Berufsverband der Augenärzte Deutschlands e.V. und die Deutsche Ophthalmologische Gesellschaft erklären, dass die Autoren keine Interessenkonflikte haben.

Dieser Beitrag beinhaltet keine von den Autoren durchgeführten Studien an Menschen oder Tieren.
Diese Stellungnahme erscheint ebenfalls in der Zeitschrift Klinische Monatsblätter für Augenheilkunde, Georg Thieme Verlag, Stuttgart. 